\title{
Functional Organization of Envelope-Responsive Neurons in Early Visual Cortex: Organization of Carrier Tuning Properties
}

\author{
Guangxing Li and Curtis L. Baker Jr \\ McGill Vision Research Unit, Department of Ophthalmology, McGill University, Montreal, Quebec, Canada H3A 1A1
}

It is well established that visual cortex neurons having similar selectivity for orientation, direction of motion, ocular dominance, and other properties of first-order (luminance-defined) stimuli are clustered into a columnar organization. However, the cortical architecture of neuronal responses to second-order (contrast/texture-defined) stimuli is poorly understood. A useful second-order stimulus is a contrast envelope, consisting of a finely detailed pattern (carrier) whose contrast varies on a coarse spatial scale (envelope). In this study, we analyzed the cortical organization of carrier tuning properties of neurons, which responded to contrast-modulated stimuli. We examined whether neurons tuned to similar carrier properties are clustered spatially and whether such spatial clusters are arranged in columns. To address these questions, we recorded single-unit activity, multiunit activity, and local field potentials simultaneously from area 18 of anesthetized cats, using single-channel microelectrodes and multielectrode arrays. Our data showed that neurons tuned to similar carrier spatial frequency are distributed in a highly clustered manner; neurons tuned to similar carrier orientation are also significantly clustered. Neurons along linear arrays perpendicular to the brain surface always exhibited similar optimal carrier spatial frequency, indicating a columnar organization. Multi-pronged tetrode recordings indicated that the diameter of these columns is $\geq 450$ $\mu \mathrm{m}$. Optimal carrier orientation was also significantly clustered but with finer-grain organization and greater scatter. These results indicate a fine anatomical structure of cortical organization of second-order information processing and suggest that there are probably more maps in cat area 18 than previously believed.

\section{Introduction}

A common anatomical feature of the cerebral cortex is columnar organization, in which neurons having similar functional properties are clustered in elongated domains perpendicular to the cortical surface (Mountcastle, 1997). In the visual cortex of cats and monkeys, several stimulus attributes exhibit columnar organization, including orientation, direction of motion, and disparity (Hubel and Wiesel, 1974; Albright et al., 1984; DeAngelis and Newsome, 1999). Most previous demonstrations of columnar or patchy organization in visual cortex have used simple stimuli composed of luminance-defined ("first-order") contours, such as sine-wave gratings (Fig. 1 $A, B$ ). However, very little is known about the anatomical representation of "second-order" contours defined by texture or contrast differences (Fig. 1C,D).

Many neurons in cat A18 respond selectively to both luminance- and contrast-defined stimuli (Zhou and Baker, 1994; Tanaka and Ohzawa, 2006; Rosenberg and Issa, 2011), the latter consisting of a finely detailed pattern (carrier) whose contrast

Received Sept. 12, 2011; revised Feb. 28, 2012; accepted April 8, 2012.

Author contributions: G.L. and C.L.B. designed research; G.L. and C.L.B. performed research; G.L. and C.L.B. contributed unpublished reagents/analytic tools; G.L. analyzed data; G.L. and C.L.B. wrote the paper.

This work was supported by Canadian Institutes of Health Research Grant MOP-9685 (C.L.B.). We thank Vargha Talebi, Amol Gharat, and Lynda Domazet for assistance with the experiments.

Correspondence should be addressed to Guangxing Li, McGill Vision Research, 687 Pine Avenue West, H4-14, Montreal, Quebec, Canada H3A 1A1. E-mail: guang.x.li@mail.mcgill.ca.

DOI:10.1523/JNEUROSCI.4662-11.2012

Copyright $\odot 2012$ the authors $\quad 0270-6474 / 12 / 327538-12 \$ 15.00 / 0$ varies on a coarse spatial scale (envelope). Neurons responding to such contrast envelopes are selective for envelope properties (orientation, spatial frequency) and also for spatial frequency and orientation of the carrier (Mareschal and Baker, 1998a, 1999). The preferred spatial frequency and orientation for contrast envelopes are similar to those for luminance gratings (Zhou and Baker, 1994; Mareschal and Baker, 1998b), with "spiral pinwheel" maps for envelope orientation (Zhan et al., 2006). However, there is no fixed relationship between tuning to carrier and luminance gratings (Mareschal and Baker, 1999), and optical imaging fails to reveal maps for carrier orientation (Zhan et al., 2006). However, the limited spatial resolution and sensitivity of optical imaging may have prevented detection of fine-grain organization for carrier properties.

If carrier parameters were clustered, it would open novel possibilities of laminar differentiation, columnar organization, and spatial maps. If these additional stimulus parameters were spatially mapped, future imaging studies could provide fresh opportunities to test theoretical ideas about limits on the number of mapped parameters (Swindale, 2000), how the mapping of an additional parameter constrains other maps (Durbin and Mitchison, 1990; Swindale, 2004), and "wiring optimization" (Chklovskii and Koulakov, 2004).

Here we recorded simultaneously from pairs of nearby single units (SUs), multiunit (MU) activity, and local field potentials (LFPs) using single-channel microelectrodes and multielectrode arrays. We ascertained whether carrier tuning in cat A18 is spa- 

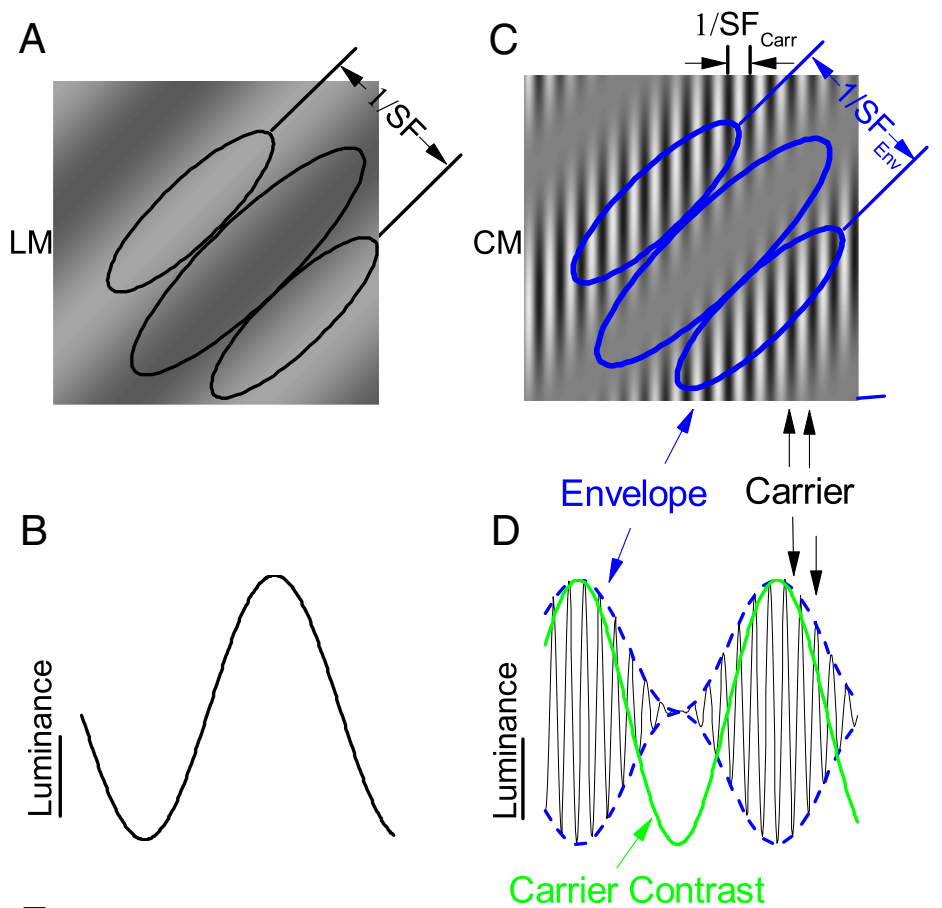

E

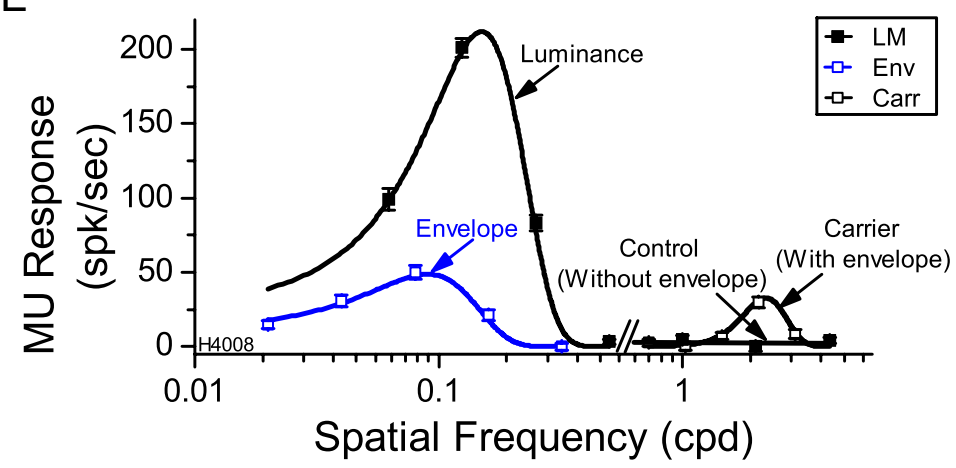

Figure 1. An example of LM (first-order) and CM (second-order) stimuli and MU response. $\boldsymbol{A}$, One frame of a luminance sine-wave grating with a superimposed diagram receptive field. $\boldsymbol{B}$, Luminance profile of the luminance sine-wave grating. $\boldsymbol{C}$, One frame of a CM grating. The carrier (Carr) is vertical with a high spatial frequency, and the envelope (Env) is oriented at $45^{\circ}$ with a lower spatial frequency. $\boldsymbol{D}$, Luminance (black) and contrast (green) profiles of the $\mathrm{CM}$ grating. $\boldsymbol{E}, \mathrm{MU}$ activity of luminance responses (filled symbols) and CM responses (opened symbols). Envelope temporal frequency, $2 \mathrm{~Hz}$; envelope orientation, $270^{\circ}$; carrier contrast, $80 \%$; carrier orientation, $270^{\circ}$; luminance sine-wave grating temporal frequency, $3 \mathrm{~Hz}$; luminance sine-wave grating contrast, $30 \%$ (control contrast, $80 \%$ ); luminance sine-wave grating orientation, $270^{\circ}$. Note that the $\mathrm{MU}$ activity at the optimal carrier frequency to the $\mathrm{CM}$ gratings (with envelope) was significantly greater than the measured response to luminance gratings (without envelope) at the same frequency. Here and in subsequent figures, spontaneous activity has been subtracted from the responses, and error bars indicate SES.

tially clustered and, if so, the spatial extent of clustering both perpendicular to, and along, the cortical surface. If there is local patch-wise clustering of carrier properties, we should find nearby neurons with highly similar tuning (DeAngelis et al., 1999) and similarly tuned local population signals (DeAngelis and Newsome, 1999; Nauhaus and Ringach, 2007).

Our results reveal an elaborate functional organization, in which neurons tuned to similar carrier spatial frequency are highly clustered and organized in columns $\geq 450 \mu \mathrm{m}$ in diameter, whereas neurons tuned to similar carrier orientation form smaller miniclusters $<250 \mu \mathrm{m}$. The organization of carrier tuning properties within A18 implies that there are more maps in this brain area than previously thought and raises theoretically important questions about the nature of these maps.

\section{Materials and Methods}

Animal preparation and maintenance. Acute experiments were performed with 12 adult cats $(2.5-4.5 \mathrm{~kg})$ of either sex. All surgical and experimental procedures were in accordance with the guidelines and policies of the Canadian Council on Animal Care and were approved by the Animal Care Committee of McGill University. Animals were initially anesthetized with isoflurane (3-5\%, in oxygen) for venous cannulation, followed by propofol (6 $\mathrm{mg} \cdot \mathrm{kg}^{-1} \cdot \mathrm{h}^{-1}$, i.v.) during subsequent surgery. All surgical wounds were infused with $0.5 \%$ bupivacaine, the corneas were protected with topical carboxymethylcellulose (1\%), the heart rate was monitored (Vet/Ox Plus 4700), and the rectal temperature was kept at $37.5^{\circ} \mathrm{C}$ (40-90-8D; FHC). After intubation or tracheal cannulation, the animal was mounted in a stereotaxic frame, and a craniotomy (A3/L4, $3 \times 3$ $\mathrm{mm}$ ) exposed area 18 (Tusa et al., 1979). To provide entry of microelectrodes, a small incision was made in the dura, which was subsequently protected with $2 \%$ agarose (Sigma) and petroleum jelly. After surgery, the animal was anesthetized and paralyzed with a continuous infusion of propofol $\left(5.3 \mathrm{mg} \cdot \mathrm{kg}^{-1} \cdot \mathrm{h}^{-1}\right.$, i.v.), fentenyl $(7.4$ $\mu \mathrm{g} \cdot \mathrm{kg}^{-1} \cdot \mathrm{h}^{-1}$, i.v.), and gallamine triethiodide $\left(10 \mathrm{mg} \cdot \mathrm{kg}^{-1} \cdot \mathrm{h}^{-1}\right.$, i.v.), supplemented with a mixture of $\mathrm{O}_{2} / \mathrm{N}_{2} \mathrm{O}$ (30:70). Vital life signs, including heart rate, EEG, and blood oxygen level, were closely monitored throughout the experiment. Expired $\mathrm{CO}_{2}$ was maintained close to 32 $\mathrm{mmHg}$, and the rectal temperature was kept at $37.5^{\circ} \mathrm{C}$. Glycopyrrolate $(0.005 \mathrm{mg} / \mathrm{kg}$, i.m. $)$ and dexamethasone $(0.6 \mathrm{mg}$, i.m. ) were administered every $12 \mathrm{~h}$ throughout the experiment. Phenylephrine hydrochloride $(2.5 \%)$ was topically applied to retract the nictitating membranes, and the pupils were dilated with $1 \%$ atropine sulfate. The optic disk was backprojected to a plotting screen (Fernald and Chase, 1971), to ascertain the locations of the area centralis of each eye (Nikara et al., 1968). Contact lenses were fitted to protect the corneas, and artificial pupils ( $2.5 \mathrm{~mm}$ diameter) were placed immediately in front of the contact lenses to further improve optical quality. Refraction with a slit retinoscope was used to select spectacle lenses to ensure that the eyes were focused on the stimulus plane at a viewing distance of $57 \mathrm{~cm}$.

Visual stimuli. Computer-controlled visual stimuli were displayed on a CRT monitor $\left(640 \times 480\right.$ pixels, $75 \mathrm{~Hz}, 36 \mathrm{~cd} / \mathrm{m}^{2}$ mean luminance; FP1350; NEC), placed at a $57 \mathrm{~cm}$ distance. Software for stimulus generation and online data analysis was custom written in MATLAB (MathWorks) using Psychophysics Toolbox (PTB-3) extensions (Brainard, 1997; Pelli, 1997; Kleiner et al., 2007), running on a Macintosh computer (Mac Pro, 2.66 GHz Quad Core Intel Xeon, 6 GB, NVIDIA GeForce GT 120; Apple Computers). Luminance nonlinearities of the CRT were corrected with an inverse gamma lookup table derived from careful calibration with a photometer (S370; United Detector Technology). Two kinds of visual stimuli were used: drifting sine-wave gratings [luminance-modulated (LM); Fig. 1 $A, B$ ] and contrast-modulated gratings (CM; Fig. 1C,D) (Zhou and Baker, 1993, 1996; Mareschal and Baker, 1998a). For sine-wave gratings, the Michaelson contrast was set at 30\%; for CM gratings, the contrast of a stationary fine-scale "carrier" grating was modulated (from 0 to $70 \%$ ) by a low spatial frequency drifting "en- 
A<smiles>[M]C1CCCCC1</smiles>

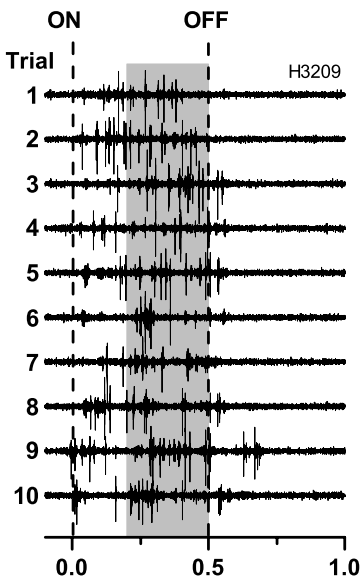

Time (s)

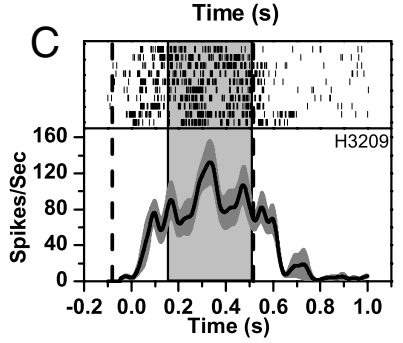

B
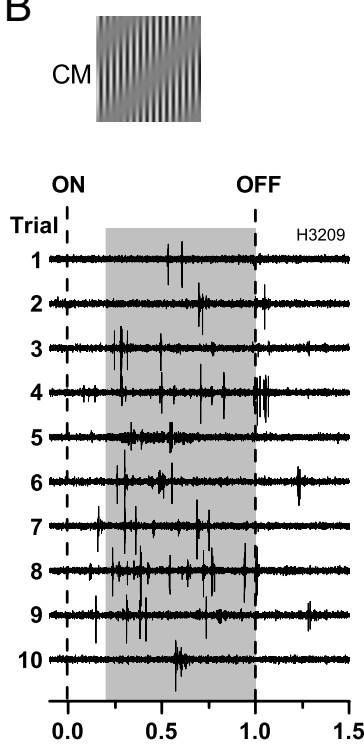

Time (s)

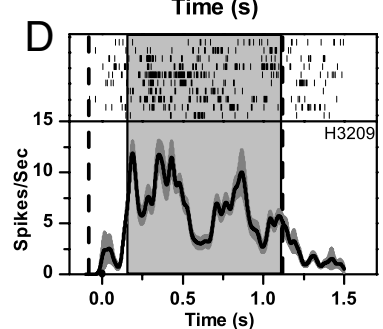

E
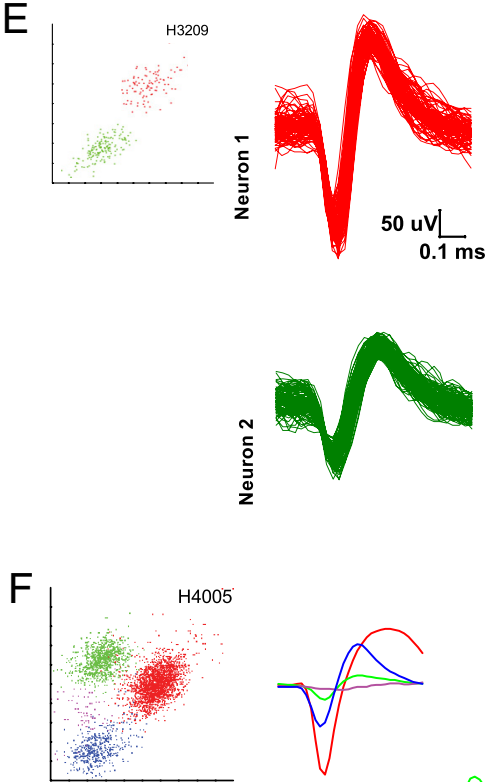

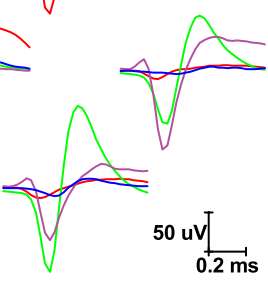

Figure 2. Examples of spike activity. Separation of MU activity and spike waveforms from pairs of SUs are illustrated for two recording sites. $\boldsymbol{A}, \boldsymbol{B}$, Raw spike traces (filtered $300-8 \mathrm{kHz}$ ) for 10 single trials, in response to LM $(\boldsymbol{A})$ and $C M(\boldsymbol{B})$ grating stimuli. $\boldsymbol{C}, \boldsymbol{D}$, Raster and mean spike density plots (15 ms Gaussian convolution, SE indicated as dark gray shading) for MU activity. Vertical dashed lines indicate onset and offset of visual stimulation. Light gray shading indicates analysis window (200 ms to offset). For both kinds of stimuli, during the stimulation period, clear spike activity can be seen. $\boldsymbol{E}$, Spike sorting result from a tungsten electrode recording. Scatter plot of spike waveform principal component analysis (PCA) components (left) shows two well-isolated SUs. Spike waveforms of the two separated SUs are shown in the right. $\boldsymbol{F}$, Spike sorting result from a tetrode array recording. Spike waveforms of four separated SUs were isolated by comparing the spike waveforms from different sites (right). The PCA scatter plot (left) shows these four well-isolated neurons.

velope" grating (Fig. 1C,D). A uniform mean luminance gray screen was displayed during all intertrial intervals and also used as a "blank" condition to measure spontaneous responses.

Data collection and experiment protocol. Tungsten microelectrodes (FHC), $1 \times 16$ linear arrays (A1x16-5mm-150-413, A1x16-5mm-150177; NeuroNexus) and $4 \times 1$ tetrode arrays (A4x1-tet-3mm-150-312; NeuroNexus) were used in these experiments. Electrode signals passed through a headstage amplifier (20×; HST/8o50-G20-GR; Plexon) and then a preamplifier $(1000 \times, 3 \mathrm{~Hz}$ to $8 \mathrm{kHz}$; PBX2/16WB-G50; Plexon). The electrode signals as well CRT timing data from a photocell (TSL12S; Texas Advanced Optoelectronic Solutions) were digitized (40 kHz, 12 bits; PCI-6071E; National Instruments) under control of Plexon Recorder software (version 2.3.2; Plexon) and saved for additional offline data analysis. When multielectrode arrays were used, one recording site was also sent to an audio monitor, window discriminator, and oscilloscope for conventional online spike isolation and was analyzed online (poststimulus time histograms and tuning curves) for guidance of the subsequent protocol.

The approximate receptive field position, optimal orientation, and dominant eye were initially determined using manually controlled search stimuli (oriented bars). All subsequent visual stimuli were delivered monocularly through the dominant eye. Neurons were then characterized quantitatively using computer-controlled stimuli, in a series of "tuning curve" measurements with randomized ordering of the varied parameters, and each set of conditions was repeated 10-20 times. Optimal stimulus parameters for drifting luminance gratings were first measured to obtain the orientation, spatial frequency, temporal frequency, and size of the best grating of the neuron. Then contrast envelope stimuli with a series of carrier spatial frequencies were tested, with the envelope spatial frequency and orientation fixed at or near their optimal values for luminance gratings (Mareschal and Baker, 1998a,b). Neurons were considered as CM responsive and included in subsequent experiments only if their responses were significantly bandpass tuned to the spatial frequency of the carrier (Song and Baker, 2007), with an optimum that was beyond the measured luminance pass band of the neurons. Note that a drifting grating (without an envelope) could not drive a response at such a high spatial frequency (Fig. $1 E$, Control). Carrier orientation tuning was then measured using the optimized carrier spatial frequency.

Signal processing. Spike and LFP signals were extracted from raw broadband data acquired by the Plexon Recorder. For spike activity, the raw data were filtered by a four-pole high-pass Butterworth digital filter ( $300 \mathrm{~Hz}$ cutoff) and then thresholded at $3 \mathrm{SD}$ to extract MU activity (Fig. $2 A-D)$. SU isolation was achieved by offline classification of spike waveforms using Offline-Sorter (version 2.8.8; Plexon) software. Sorting was initiated by the "semi-automatic $K$-means" algorithm, based on a $2 \mathrm{D}$ or $3 \mathrm{D}$ feature space with the initial cluster centers selected manually. SUs were further isolated carefully using a manual clustering procedure, which used additional 2D/3D feature spaces, autocorrelograms, crosscorrelograms, and refractory periods. Only clearly delineated clusters of spikes that were well separated from background noise were accepted as SUs (Fig. 2E,F).

For LFP activity, the raw data were low-pass filtered and then downsampled to $2 \mathrm{kHz}$ (Fig. $3 A, B$ ). Thus, the frequency pass band for LFP activity was $\sim 3-300 \mathrm{~Hz}$ as a result of the recording system and the digital filtering. In this paper, we only analyzed LFPs in their sustained period, i.e., from $200 \mathrm{~ms}$ after stimulus onset to the end of the stimulus (Siegel 
A
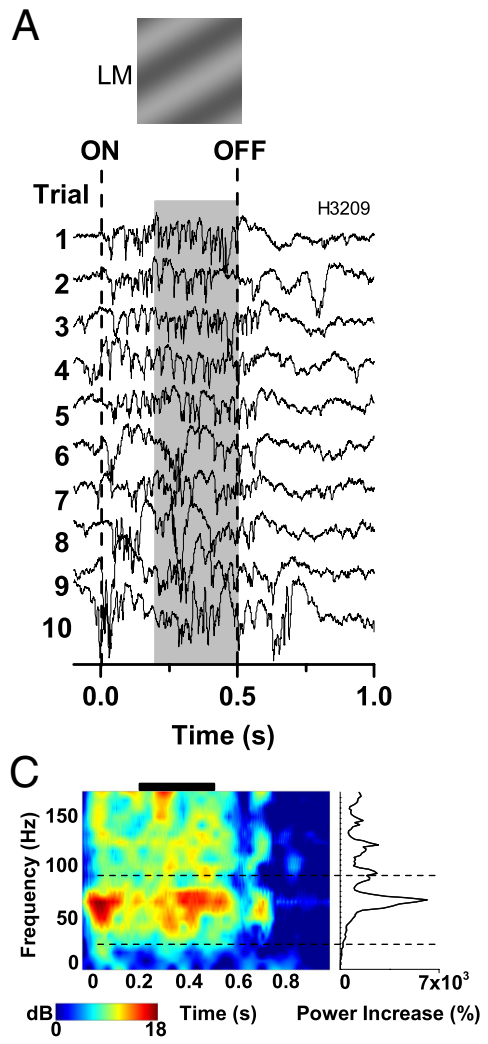

B
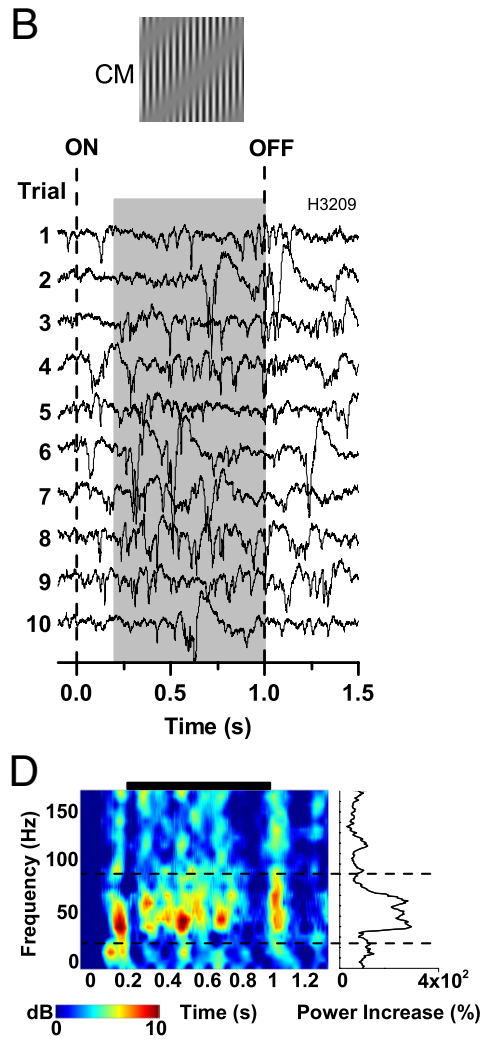

width at half-maximal height $\left(\mathrm{SF}_{\mathrm{bw}}\right)$, in cycles per degree (cpd), was calculated as $1.65 \alpha$ and subsequently converted to octaves.

The orientation tuning was characterized as orientation bias (OB) (Wörgötter and Eysel, 1987; Leventhal et al., 2003), which is a complex number incorporating all of the data points on the tuning curve within a vector summation:

$$
\mathrm{OB}=\frac{\sum_{\mathrm{k}} \mathrm{R}_{\mathrm{k}} \mathrm{e}^{\mathrm{i} 2 \theta_{\mathrm{k}}}}{\sum_{\mathrm{k}} \mathrm{R}_{\mathrm{k}}},
$$

where $R_{k}$ is the baseline subtracted mean response to a stimulus with orientation $\theta_{k}$ in radians. The magnitude, $|\mathrm{OB}|$, provides a bounded range from 0 to 1 , with 0 indicating complete insensitivity to orientation (anisotropy) and unity corresponding to response at only one orientation. Cells were considered as clearly orientation tuned if $|\mathrm{OB}|>0.3$ (Yao and Dan, 2001). The angle, $\overrightarrow{\mathrm{OB}}$, provides the optimal orientation.

The degree of clustering for carrier properties was quantified by the method of DeAngelis et al. (1999):

$$
\text { Clustering Index }=\frac{\tilde{X}_{\text {Grand Random }}}{\tilde{X}_{\text {Paired }}} .
$$

Figure 3. Examples of LFP activity. LFP responses to LM (left column) and CM (right column) grating stimuli. $A, B$, LFP traces (filtered 3-300 Hz) for 10 single trials, extracted from the same recordings as Figure 2. Vertical dashed lines indicate onset and offset of visual stimulation, and gray areas indicate analysis window (200 ms to offset). C, D, LFP spectrograms for LM and CM stimuli. Black horizontal bars at top of each panel indicate temporal analysis window ( $200 \mathrm{~ms}$ to offset). Horizontal dashed lines indicate gamma band $(25-90 \mathrm{~Hz})$. For both kinds of stimuli, during the stimulation period, clear LFP activity can be seen, with a prominent increase in power in the gamma band.

Here $\tilde{X}_{\text {Paired }}$ denotes the median distance of pairs in a scatter plot from the equality line:

$$
\tilde{X}_{\text {paired }}=\operatorname{Median}\left(\frac{\left|x_{i}-y_{i}\right|}{\sqrt{2}}\right) \quad i=1,2,3 \ldots,
$$

and König, 2003; Kayser and König, 2004; Berens et al., 2008; Gieselmann and Thiele, 2008), indicated in gray in Figure 3, $A$ and $B$. LFP signal processing was performed using an open-source MATLAB toolbox, Chronux 2.0 (Bokil et al., 2010). Residual $60 \mathrm{~Hz}$ line noise was removed using a regression method (Thomson, 1982), and LFP time-spectral histograms (Fig. $3 C, D$ ) were estimated using a multitaper approach (Thomson, 1982) with "Slepian functions" (Slepian and Pollack, 1961; Henrie and Shapley, 2005; Mitra and Bokil, 2008). A spectral band of $\pm 5 \mathrm{~Hz}$ was analyzed using four Slepian tapers (500 ms data analysis window). The LFP spectra were normalized and expressed as a percentage change from baseline (Henrie and Shapley, 2005):

$$
\Delta R(f)=\left(S(f) / S_{\mathrm{bl}}(f)-1\right) \times 100 \%,
$$

where $\Delta R$ denotes the power increase at frequency $f, S(f)$ denotes the mean power for frequency $f$ (estimated from a given stimulus condition), and $S_{\mathrm{bl}}(f)$ denotes the mean power for frequency $f$ (estimated from blank conditions). The LFP response to a given stimulus condition was taken as $\Delta R$ averaged over the gamma band $(25-90 \mathrm{~Hz})$, which has been widely studied (Pesaran et al., 2002; Henrie and Shapley, 2005; Viswanathan and Freeman, 2007; Katzner et al., 2009) and found to be highly tuned to visual stimuli (Frien et al., 2000; Henrie and Shapley, 2005; Liu and Newsome, 2006; Berens et al., 2008; Khayat et al., 2010).

Data analyses. Subsequent analyses were applied in the same manner to SU, MU, and LFP responses. Spatial frequency response data were fit with a Gaussian function (DeAngelis et al., 1994):

$$
R(\mathrm{sf})=k \times e^{-\left(\left(\mathrm{sf}^{-} \mathrm{SF}_{\mathrm{opt}}\right) / \alpha\right)^{2}}+R_{0},
$$

where $k, \mathrm{SF}_{\mathrm{opt}}, \alpha$, and $R_{O}$ are free parameters, sf denotes spatial frequency, and $\mathrm{SF}_{\mathrm{opt}}$ denotes optimal spatial frequency. The spatial frequency band- where $x_{i}$ and $y_{i}$ denote the values of a carrier property in the $i$ th cell pair. $\tilde{X}_{\text {Grand Random }}$ denotes the median of the distribution of random medians $\tilde{X}_{\text {Random_Paired }}$, which is similar to $\tilde{X}_{\text {Paired }}$ but with paired datasets created by random resampling:

$$
\begin{aligned}
& \tilde{X}_{\text {Grand Random }} \\
& =\operatorname{Median}(\underbrace{\left.\tilde{X}_{\text {Random_Paired }}, \tilde{X}_{\text {Random_Paired }}, \ldots, \tilde{X}_{\text {Random_Paired }}\right)}_{N=5000} .
\end{aligned}
$$

A clustering index of unity indicates no clustering, and larger values indicate a greater degree of clustering.

Statistical significance of the clustering index was determined by the proportion of $\tilde{X}_{\text {Random_Paired }}$ having a value less than $\tilde{X}_{\text {Paired }}$ (e.g., $p=$ 0.001 indicates that $\tilde{X}_{\text {Random_Paired }}$ was less than $\tilde{X}_{\text {Paired }}$ in 5 of 5000 bootstrap samples).

\section{Results}

The data analyzed here were from 204 recording sites in 12 cats using tungsten electrodes (66 sites), linear arrays (104 sites), and tetrode arrays (34 sites). From each site, we recorded spike activity and LFPs simultaneously.

\section{Electrophysiological signals}

Exemplar spike traces from a single recording site during stimulation with optimized luminance and CM gratings are shown in Figure 2, $A$ and $B$. The visual stimulus appeared and started drift- 
ing at $0 \mathrm{~s}$ and lasted $0.5 \mathrm{~s}$ for luminance stimuli and $1.0 \mathrm{~s}$ for $\mathrm{CM}$ stimuli. MU activity is shown as raster plots and spike density as a function of time in Figure 2, C and $D$. The CM responses were generally weaker than those to luminance gratings, consistent with previous findings (Zhou and Baker, 1993, 1996; Tanaka and Ohzawa, 2006; Song and Baker, 2007), but the induced spike activity was readily visible in the CM traces (Fig. $2 B, D$ ). To make the results comparable with LFP data (below), we only included the stimulation period from $200 \mathrm{~ms}$ after stimulus onset in the following analysis. Samples of isolated SUs from a tungsten electrode and a tetrode array are shown in Figure 2, $E$ and $F$. From the recording site with the tungsten electrode (Fig. 2E), we separated two SUs, which served as one pair of cells in subsequent analyses. For the tetrode array site (Fig. $2 F$ ), four SUs were isolated, providing a total of six pairs. One neuron (purple), which had a very low firing rate, was luminance responsive but not CM responsive (shown in Fig. $4 D$, purple), so it only exhibited spontaneous activity when responding to $\mathrm{CM}$ stimuli.

Figure $3, A$ and $B$, shows LFP traces recorded during the same 10 trials as the spikes in Figure 2, $A$ and $B$. Note the fast oscillations that increased noticeably during the sustained period. The enhancement of amplitudes and higher regularity of the oscillations shortly after stimulus onset was a general observation in these recordings, similar to previous results (Berens et al., 2008; Gieselmann and Thiele, 2008). The LFP response has been characterized by two components: a transient response ("visual evoked potential") and sustained oscillations that emerge in the later part of the LFP response. Here we only consider the sustained responses, by restricting the analysis to the period starting $200 \mathrm{~ms}$ after stimulus onset. Spectrograms of the LFP signals (Fig. 3C,D) showed marked increases in signal power across the gamma band, here chosen from 25 to $90 \mathrm{~Hz}$ (Henrie and Shapley, 2005; Viswanathan and Freeman, 2007; Katzner et al., 2009), and this band was analyzed in the following results.

\section{Carrier spatial frequency tuning}

Figure 4 depicts CM responses from a tungsten electrode (left column) and a tetrode array recording (right column) as functions of carrier spatial frequency, using an envelope orientation fixed at the value that was optimal for luminance gratings. The carrier spatial frequency responses were bandpass, consistent with previous studies for SUs (Zhou and Baker, 1996; Tanaka and Ohzawa, 2006; Song and Baker, 2007; Rosenberg and Issa, 2011). In most of our recording sites, the increase in LFP gamma-band power was also clearly tuned to the carrier spatial frequency of the CM stimulus (Fig. 4C,F). We compared the preferred frequency and bandwidth of the tuning functions for nearby SU, MU, and
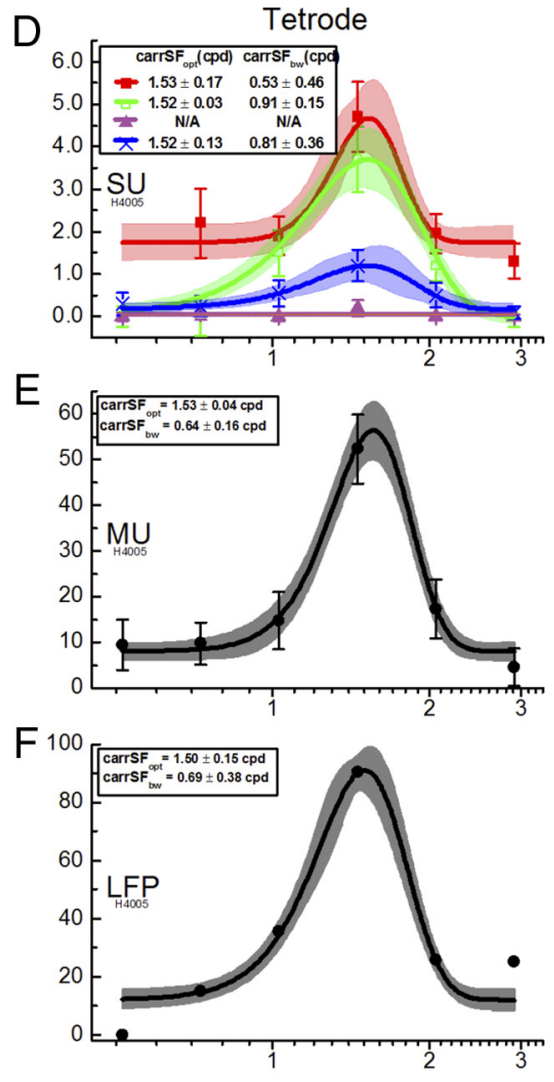

\section{Carrier Spatial Frequency (cpd)}
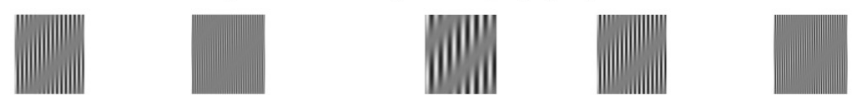

Figure 4. Examples of carrier spatial frequency tuning data. Each column shows simultaneously recorded SU, MU, and LFP tuning curves for carrier spatial frequency from a tungsten electrode (left column) and a tetrode array (right column), with superimposed Gaussian curve fits. $A$, SU carrier (carr) spatial frequency tuning curves for two isolated nearby SUs sorted from a the isolation of these two SUs is shown in Figure 2E. B, MU tuning curve extracted from the same recording site as $\boldsymbol{A}$. C, LFP tuning curve extracted from the same recording site as $A . D, S U$ carrier spatial frequency tuning curves of extred nearby SUs recorded from a tetrode array. The isolation of these four SUs is shown in Figure 2F. E, MU tuning curve optimal carrier spatial frequency tuning curves extracted from SU, MU and LEP pairs always show similar optimal frequency, but not necessarily similar tuning bandwidths, for that recording site. Shaded areas indicate $\pm 1 \mathrm{SD}$, estimated by bootstrapping $\left(n_{\text {boot }}=\right.$ 1000). Estimates of optimal spatial frequency and bandwidth are shown as mean \pm SD.

LFP signals that were simultaneously recorded from the same site. A Gaussian fit (see Materials and Methods) to each tuning curve provided estimates of the optimal carrier spatial frequency $\left(\mathrm{SF}_{\mathrm{opt}}\right)$ and tuning bandwidth $\left(\mathrm{SF}_{\mathrm{bw}}\right)$, with a bootstrap resampling method (Efron and Tibshirani, 1993) used to estimate fitting errors. We found that nearby single neurons (Fig. $4 A, D$; also shown in Fig. 2E, $F$ ) typically showed very similar optimal carrier spatial frequency but some degree of variation in tuning bandwidth. One of the neurons (Fig. 4D, purple) showed no response to $\mathrm{CM}$ stimuli but clear response to luminance gratings (data not shown), suggesting that the neurons nearby to a given CM-responsive neuron are not necessarily also CM responsive.

If nearby neurons have similar optimal spatial frequencies, then local population signals (MU and LFP) from the same recording site should exhibit bandpass tuning and have similar optimal carrier spatial frequencies as the SUs. To compare carrier spatial frequency dependence of MU and LFP responses with that of SU data, we plotted optimal carrier spatial frequencies for MU (Fig. $4 B, E$ ) and LFP (Fig. 4C,F) signals against those for SUs 

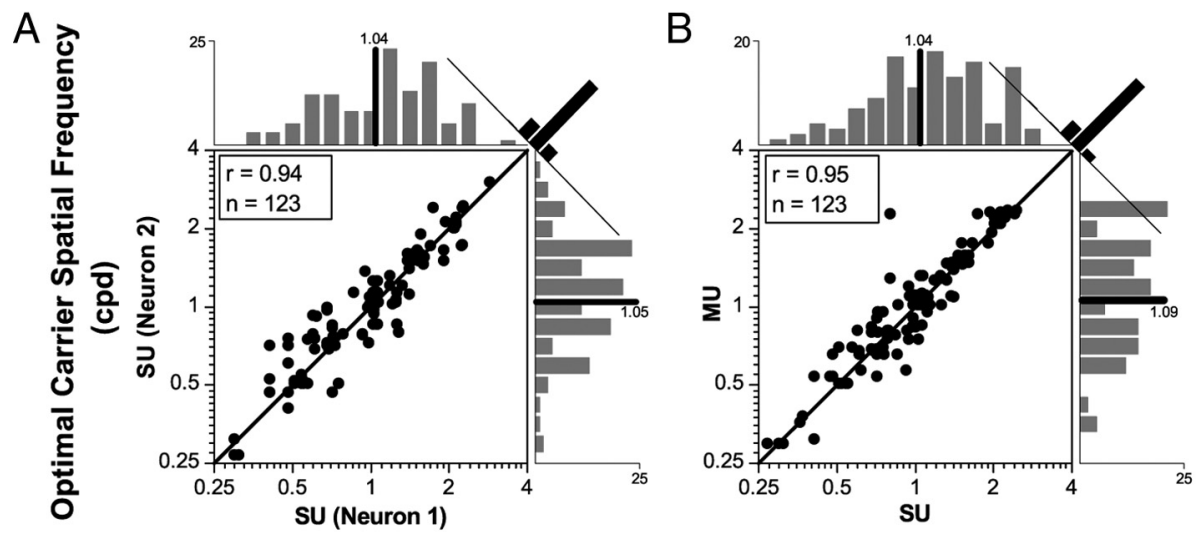

Optimal Carrier Spatial Frequency (cpd)

D

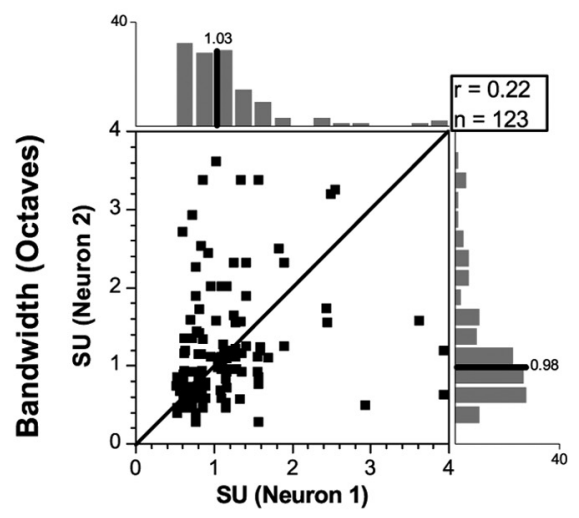

$E$

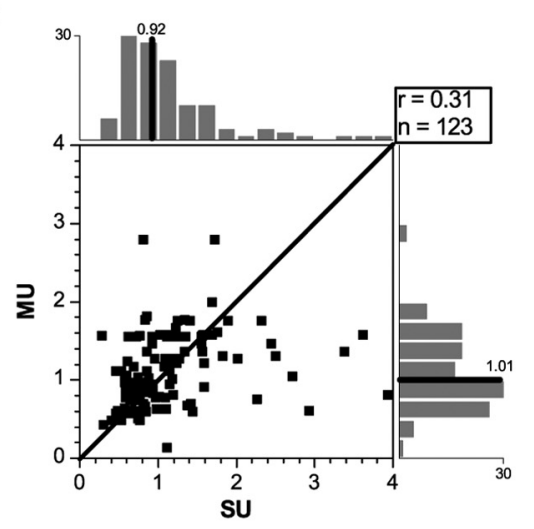

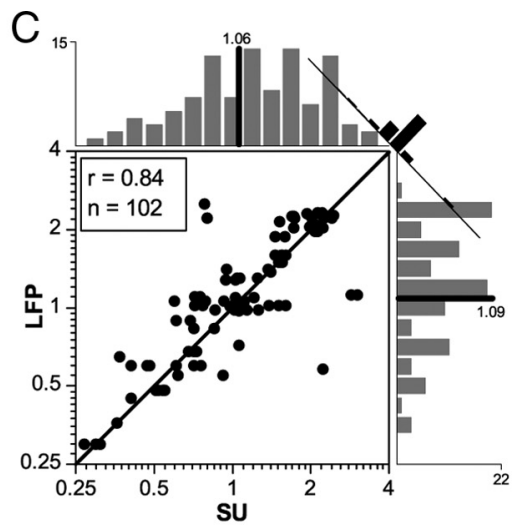

$\mathrm{F}$

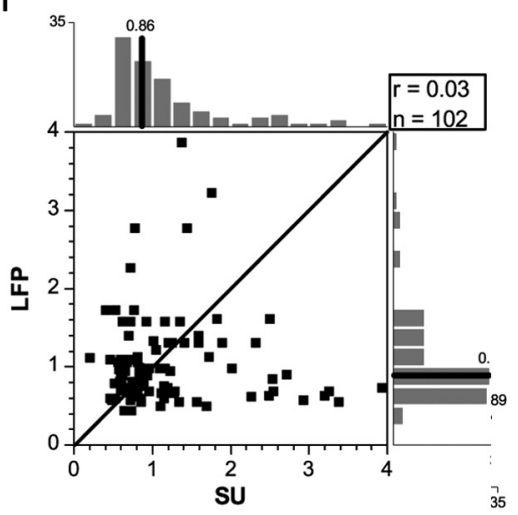

Bandwidth (Octaves)

Figure 5. Comparison of carrier spatial frequency tuning properties of simultaneously recorded SU, MU_SU, and LFP_SU pairs. In each scatter plot, the parameter value for one SU is plotted on the horizontal axis, and the value for a simultaneously recorded nearby SU, MU, or LFP response is plotted on the vertical axis. Diagonal lines indicate 1:1 ratio. $A, 0$ ptimal carrier spatial frequencies of nearby neurons show strong clustering, with most data points distributed tightly around the equality line. $B$, Optimal carrier spatial frequency of MU_SU pairs, showing strong clustering with most data points distributed tightly around the equality line. C, Pairwise comparison of optimal carrier spatial frequencies of SU and LFP responses extracted from the same recording sites. $\boldsymbol{D}$, Pairwise comparison of the tuning bandwidths of nearby neurons. $\boldsymbol{E}$, Pairwise comparison of the tuning bandwidths of $S U$ and MU signals extracted from the same recording sites. $\boldsymbol{F}$, Pairwise comparison of the tuning bandwidths of SU and LFP signals extracted from the same recording sites. Histograms at right top corners denote distribution of deviations from equality. Dark lines in marginal histograms indicate median values.

from the same recording site. Note that the optimal carrier spatial frequencies of these neurons for MU and LFP signals were similar to those for $\mathrm{SU}$ responses, but for tuning bandwidth, the $\mathrm{MU}$ data shown in Figure $4 B$ are slightly narrower than one SU (Fig. $4 A$, red) but broader than the other (Fig. $4 A$, green). A similar result can be seen in Figure 4, $D$ and $E$. For LFP data, the bandwidth in Figure $4 C$ is substantially broader than for SUs and MUs, but the data in Figure $4 F$ are narrower than for two of the SUs (green and blue) in Figure $4 D$ and is slightly broader than for the MU data.

Clustering of carrier spatial frequency tuning parameters

We confirmed this similarity across a broader sample of recording sites with scatter plots of optimal carrier spatial frequency and tuning bandwidth from simultaneously recorded SU, MU, and LFP signals (Fig. 5). This comparison only includes sites for which signals were tuned and which satisfied our criterion for clear second-order response (i.e., bandpass carrier spatial frequency tuning with a well-defined optimum).

In the scatter plot of Figure $5 A$, each plotted point indicates the optimal carrier spatial frequency for a given neuron, plotted against the corresponding value for a simultaneously recorded nearby neuron. The data points appear clustered near the 1:1 ratio line, with a very high correlation (Pearson's $r=0.94$ ) between values for the two cells of each pair. Consistent with this correlation, the clustering index was relatively high $(6.25, p<$ 0.001). The marginal histograms show distributions of optimal spatial frequencies, typically ranging from 0.5 to $2.0 \mathrm{cpd}$ (median, $1.04 \mathrm{cpd}$; mean $\pm \mathrm{SE}, 1.12 \pm 0.03 \mathrm{cpd}$ ), consistent with previous cat A18 results (Mareschal and Baker, 1999). This range of optimal frequencies was not simply a consequence of variation of retinal eccentricity: we observed a wide range of optimal carrier spatial frequencies at both low $\left(\sim 5-10^{\circ}\right)$ and high $\left(\sim 20-30^{\circ}\right)$ eccentricity, which is consistent with previous results (Zhou and Baker, 1996) that there is no clear correlation between optimal carrier spatial frequency and eccentricity. In Figure $5 B$, each plotted point indicates the optimal carrier spatial frequency of MU activity, plotted against the simultaneously recorded SUs. Consistent with this result, the optimal carrier spatial frequencies extracted from MU data (Fig. 5B) were highly correlated with SU data (Pearson's $r=0.95$ ) and strongly clustered (clustering index, 7.57; $p<0.001$ ). Moreover, the optimal carrier spatial frequencies extracted from gamma-band LFP (Fig. 5C) was also highly correlated with SU data (Pearson's $r=0.84$ ), and the data points clustered about the unity line (clustering index, 5.00; $p<0.001$ ).

Figure $5 D$ shows that the tuning bandwidth of nearby neurons was neither clustered (clustering index, $1.19 ; p=0.11$ ) nor clearly correlated (Pearson's $r=0.22$ ). The marginal histograms show 


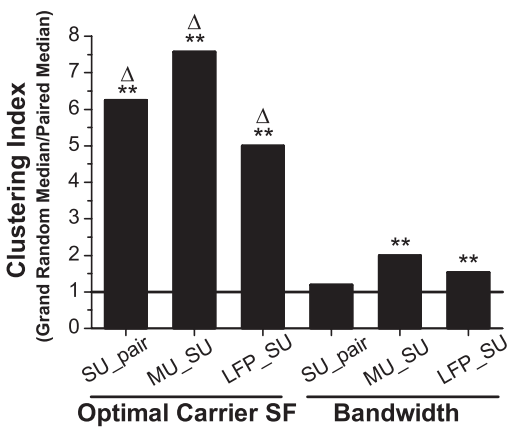

Figure 6. Quantitative comparison of clustering for carrier spatial frequency tuning. Bars indicate clustering index values (see Materials and Methods) for optimal carrier spatial frequency (left) and tuning bandwidth (right) for SU_SU, SU_MU, and LFP_SU pairs. ** indicate that clustering index is significantly $>1.0(p<0.01) . \Delta$ indicates high linear correlation (Pearson's $r>0.75$ ) across data pairs. Horizontal line for clustering index of 1.0.

distributions of tuning bandwidth typically ranging from 0.4 to 1.4 octaves (median, 1.03 octaves; mean \pm SE, $1.18 \pm 0.04$ octaves), consistent with previous cat A18 results (Mareschal and Baker, 1999). Across the sample of recording sites, the tuning bandwidth of SUs was weakly (but significantly) clustered with that of MU activity (Fig. 5E; clustering index, $2 ; p<0.001$ ) and LFP (Fig. 5F; clustering index, 1.53; $p<0.01$ ) but not clearly correlated with them (Pearson's $r=0.31$ and 0.03 , respectively). The tuning bandwidth for MU and LFP data was not significantly broader or narrower than for SU data ( $p=0.20$ and 0.52 , respectively, paired Wilcoxon's signed-rank test). This result seemed surprising because one might expect local population signals to exhibit broader tuning bandwidth than SUs. However such a result might arise from the substantial variation in strength of CM responses from cell to cell so that, in some cases, the population responses could be dominated by a subset of high firing rate neurons that had narrower tuning bandwidth than a given $\mathrm{SU}$.

A summary comparison of the degree of clustering for optimal carrier spatial frequency and tuning bandwidth is shown as a bar graph of clustering index values in Figure 6. The optimal carrier spatial frequencies exhibited strong and highly significant clustering for SU, MU_SU, and LFP_SU pairs. Each of these comparisons also showed highly significant correlation. We also observed much weaker but statistically significant clustering for MU_SU bandwidth and LFP_SU bandwidth, although the data did not show significant linear correlation.

\section{Carrier orientation tuning}

To quantitatively evaluate the carrier orientation selectivity of SU, MU, and LFP signals for CM stimuli, the envelope orientation was fixed at the measured optimum, and the orientation of the carrier was varied. Figure 7 displays polar plots of orientation response of two CM-responsive sites recorded from a tungsten electrode (top row) and a tetrode array (bottom row), from which their optimal orientation and $\mathrm{OB}$ could be compared. Figure $7 A$ shows responses of two nearby neurons, in red and green, which are tuned for carrier orientations differing by $\sim 40^{\circ}$. The tuning curve of one neuron is substantially broader than that of the other ( $\mathrm{OB}$ of 0.11 and 0.30 , respectively). This diversity in tuning bandwidth is consistent with previous results showing that some A18 neurons are highly selective for carrier orientation, whereas others are biased to various degrees or even isotropic (Mareschal and Baker, 1998a, 1999). Figure 7, B and $C$, depicts MU and LFP responses recorded from the same site. The MU and
LFP signals showed optimal carrier orientation at $95^{\circ}$ and $83^{\circ}$, respectively, which exhibited a difference of up to $48^{\circ}$ and $60^{\circ}$ from one of the SUs (green) at the same site. Both the MU and LFP signals showed poor tuning to carrier orientation $(\mathrm{OB}<$ 0.2 ). The carrier orientation response at another recording site is shown in the bottom row of Figure 7. At this site, all three isolated nearby neurons showed similar optimal carrier orientation as well as high orientation selectivity $(\mathrm{OB}>0.3$; Fig. $7 D)$. Consistent with this result, the MU response was tuned to a similar orientation as the SUs at this site, with strong orientation selectivity (Fig. 7E). However, the LFP was tuned to a slightly different orientation, with a weaker selectivity than $\mathrm{SU}$ and $\mathrm{MU}$ responses (Fig. $7 F$ ).

Thus, it appears that nearby neurons might be tuned to either similar or different carrier orientations at some sites and that highly selective neurons can be mixed with broadly tuned neurons at a given site. In addition, there were some sites at which the nearby neurons exhibited high selectivity for similar carrier orientation, but the LFP still revealed a broad tuning at a different optimal orientation.

\section{Clustering of carrier orientation tuning parameters}

To examine the results across a larger sample of recording sites, we show scatter plots of the optimal carrier orientation and carrier OB of simultaneously recorded SU, MU, and LFP data in Figures 8 and 9. In Figure $8 A$, the optimal carrier orientation of each neuron was compared with that for the second cell of each SU pair. The solid line represents equal optimal orientations, whereas the dashed lines represent orthogonal preferred orientations. The data was not tightly clustered around the equality line, revealing some variation in optimal carrier orientation between two nearby neurons. Approximately 50\% (55 of 110 pairs) of nearby neurons differed in optimal carrier orientation $<15^{\circ}$; $\sim 87 \%$ (96 of 110 pairs) differed $<45^{\circ}$. Thus, nearby neurons tended to have similar optimal carrier orientations (clustering index, 2.88; $p<0.001$ ) but were poorly correlated (Pearson's $r=$ 0.34 ; data collapsed into a $0-90^{\circ}$ range).

Before concluding that the optimal carrier orientation of nearby neurons is not well correlated, we must consider the possibility that the measured orientation differences could arise from weakly tuned or even isotropic neurons with ill-defined optima (Fig. 7A, red). To examine this possibility, in Figure $8 A$, we plotted highly selective neuron pairs $(\mathrm{OB}>0.3)$ as red squares and pairs in which one or both members were poorly selective $(\mathrm{OB}<$ 0.3 ) as black squares. Similarly, the distribution of orientation differences between SU pairs in Figure $8 B$ is shown as red bars for $\mathrm{OB}>0.3$ and black bars for $\mathrm{OB}<0.3$. The results show that the difference in optimal carrier orientation clearly exists in both highly tuned and poorly selective neuron pairs: the two distributions of the orientation difference were not significantly different ( $p=0.29$, Mann-Whitney $U$ test). Figure $8 C$ shows a scatter plot of $\mathrm{OB}$ values of nearby neurons. The data were highly scattered, without a clear linear correlation of $\mathrm{OB}$ between the nearby neurons (Pearson's $r=0.26$ ). Nevertheless, the pairs were significantly clustered (clustering index, 1.38; $p<0.01$ ), although rather more weakly than for optimal carrier orientation.

In Figure 9, $A$ and $B$, the optimal carrier orientations for MU and LFP data are plotted against those for SUs from the same recording sites. This comparison only includes sites for which the MU and LFP signals exhibited clear evoked responses. For $\sim 65 \%$ (70 of 108) of MU sites and 35\% (31 of 89) of LFP sites, the difference in optimal carrier orientations was $<15^{\circ}$; $\sim 90 \%$ (97 of 108 ) of MU sites and $82 \%$ (73 of 89 ) of LFP sites showed a differ- 
A
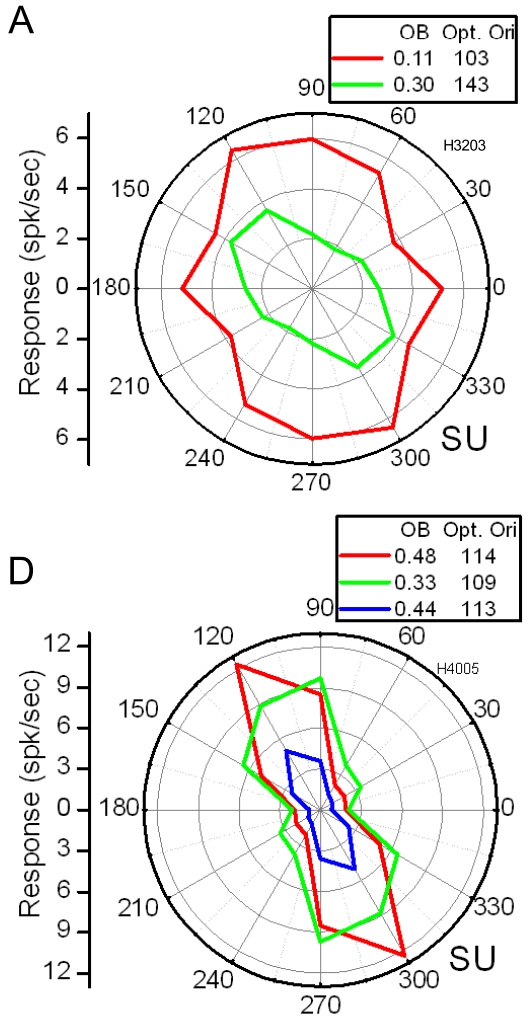

B

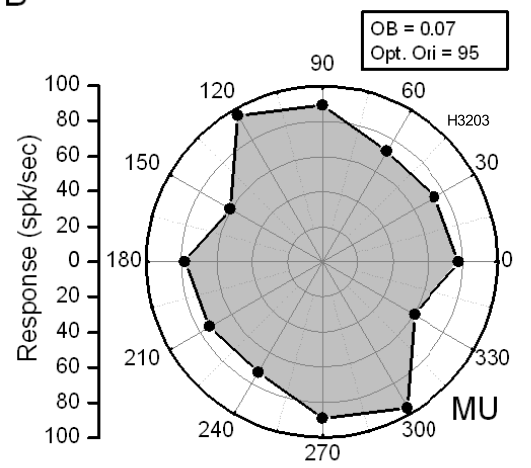

E

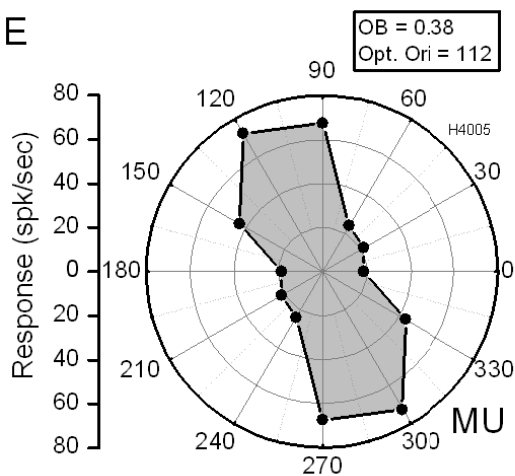

C

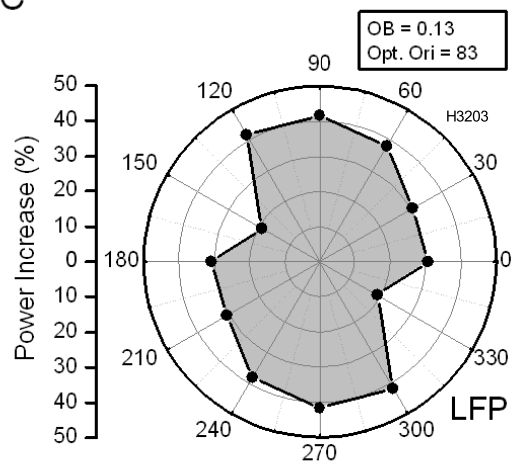

$\mathrm{F}$

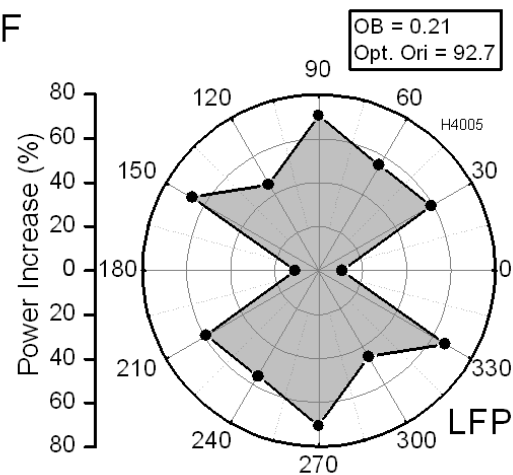

Figure 7. Examples of carrier orientation tuning curves. Simultaneously recorded SU, MU, and LFP tuning curves for carrier spatial frequency are shown from a tungsten electrode (top row) and a tetrode array (bottom row). $\boldsymbol{A}$, Carrier orientation tuning curves for two isolated SUs (red and green) recorded from a tungsten electrode. $\boldsymbol{B}$, MU tuning curve extracted from the same recording site as $A$. C, Gamma LFP tuning curve extracted from the same recording site as $A$. D, SU carrier orientation tuning curves of three isolated nearby SUs recorded from a tetrode array (data from these SUs are also shown in Figs. $2 F$ and 4). $\boldsymbol{E}$, MU tuning curve extracted from the same recording site as $\boldsymbol{D}$. F, LFP tuning curve extracted from the same recording site as $\boldsymbol{D}$.
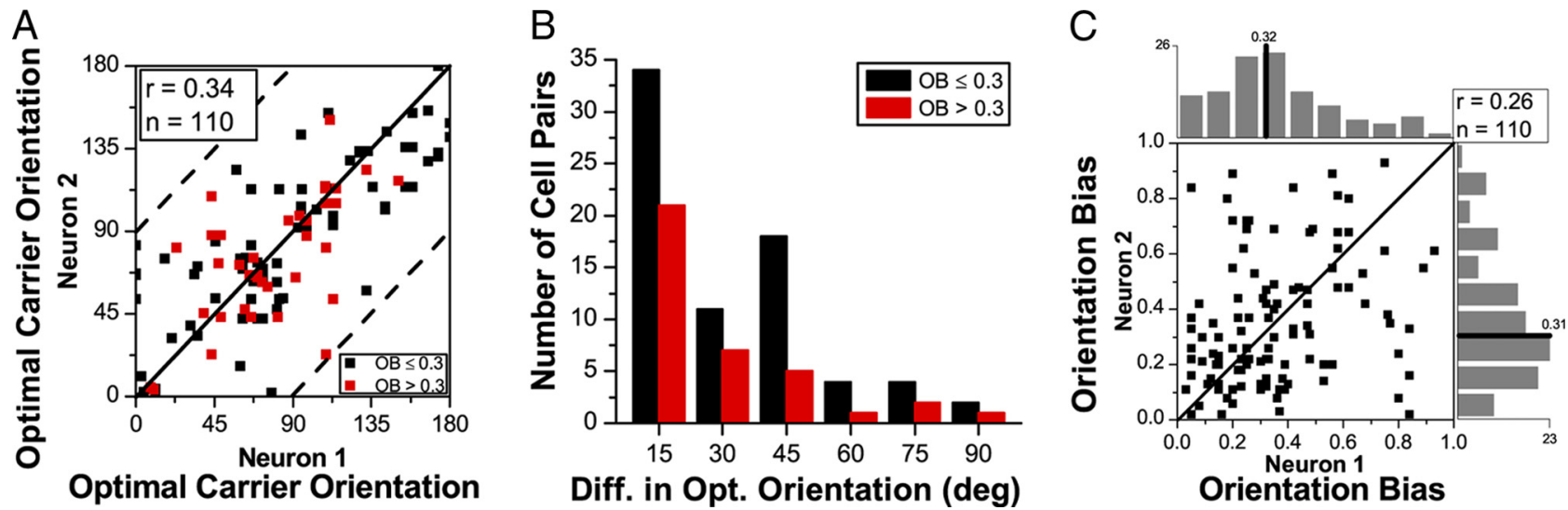

Figure 8. Comparison of carrier orientation tuning properties of nearby SUs. In each scatter plot, the parameter value for one neuron is plotted on the horizontal axis, and the value for a simultaneously recorded nearby neuron is plotted on the vertical axis. $\boldsymbol{A}$, Scatter plot of optimal carrier orientations. Because orientation is a circular variable, the largest possible difference in orientation between two neurons is $\pm 90^{\circ}$. Therefore, the plotted orientation values are "wrapped" to fall within $\pm 90^{\circ}$ of one another. The dots in red indicate pairs of more highly orientationselective SUs $(O B>0.3)$. B . Histogram of magnitude of difference between the optimal carrier orientations derived from nearby neurons. Nearby neurons tended to have somewhat similar optimal carrier orientation, but the relationship exhibited considerable scatter that was not related to tuning bandwidth. Filled bars correspond to pairs in which at least one of the neurons is poorly tuned $(O B \leq 0.3)$, and open bars represent pairs of more clearly tuned neurons $(O B>0.3)$. C, Scatter plot of $O B$ values of nearby neurons, which ranged widely from 0 (isotropic) to values approaching unity (highly tuned). Orientation tuning bandwidths were highly variable across neurons and not highly correlated in nearby neurons.

ence of $<45^{\circ}$. The clustering index as well as the correlation coefficient of MU_SU pairs (clustering index, $4.78 ; p<0.001$; Pearson's $r=0.52$ ) was higher than that of SU pairs (clustering index, 2.88; $p<0.001$; Pearson's $r=0.34$ ) and LFP_SU pairs (clustering index, 1.95; $p<0.001$; Pearson's $r=0.24$ ). Figure 9, $C$ and $D$, shows comparisons of carrier orientation selectivity, which was quantified with $\mathrm{OB}$ ranging from 0 (isotropy) to unity (sharp tuning). The OB values plotted in Figure $9 \mathrm{C}$ fell more often below the 1:1 line, indicating that the MU responses had significantly broader orientation bandwidths than the SU responses $(p<0.001$, paired Wilcoxon's signed-rank test). Furthermore, there was no clear linear correlation between the $\mathrm{OB}$ values for MU and SU responses (Pearson's $r=0.44$ ). We similarly did not find a correlation between the OB values of LFP and 
SU responses (Fig. 9D; Pearson's $r=$ 0.48 ), and again the LFP tuning was significantly broader than that for SUs $(p<$ 0.001 , paired Wilcoxon's signed-rank test).

The data in Figure 10 indicate that the degree of clustering for optimal carrier orientation was generally weaker (although still significant) than that for optimal carrier spatial frequency (Fig. 6), consistent with the lack of linear correlation for any of the parameter pairs. However, the MU_SU pairs exhibited a relatively high clustering index for optimal carrier orientation, suggesting that the cluster size for carrier orientation is comparable with the recording radius of MU activity (Liu and Newsome, 2006).

Topography of carrier tuning properties

As indicated above, the optimal carrier spatial frequencies of nearby neurons were highly correlated and clustered. Nearby neurons also tended to have similar optimal carrier orientation, and MU_SU pairs exhibited a high clustering index. Overall, these results suggested that carrier tuning properties are well organized rather than randomly distributed in the brain. To begin exploring whether optimal carrier tuning properties are organized in a columnar manner, we examined MU data from 16channel linear arrays. Figure 11 shows optimal carrier spatial frequencies (circles) and orientations (squares) from linear array recordings in penetrations perpendicular to the brain surface. Almost all of the CM-

responsive sites exhibited similar optimal carrier spatial frequencies along the extent of the arrays, with the exception of a single site (deepest in Fig. 11 A) that might have been attributable to a penetration not being perfectly aligned with the columnar architecture. Although our linear arrays did not span the full depth of the cortex, the similarity of carrier spatial frequency was confirmed for relative depths up to $\sim 2100 \mu \mathrm{m}$ (Fig. 11C). Optimal carrier orientation was somewhat more scattered, but overall still relatively constant with depth in two of three cases (Fig. 11A,B). However, in one case (Fig. $11 D)$, the preferred orientations appeared almost random. Thus, carrier spatial frequency exhibited a clear columnar organization, but the organization of carrier orientation could be more variable.

To estimate the spatial extent of carrier tuning properties across the cortical surface, we examined MU data from fourshank tetrode arrays. Figure 12 shows different examples of optimal carrier spatial frequency (circles) and orientation (squares) from recording sites at the four shanks. Sites as far apart as 450 $\mu \mathrm{m}$ along the brain surface still showed very similar optimal carrier spatial frequencies. Preferred carrier orientations, however, varied substantially from one shank to another and even among different recording sites in an individual tetrode, which are only $25 \mu \mathrm{m}$ apart. Together with the results from Figure 11, these data suggest that carrier spatial frequency is arranged in a columnar manner on a coarse scale (i.e., with a diameter $\geq 450$
B

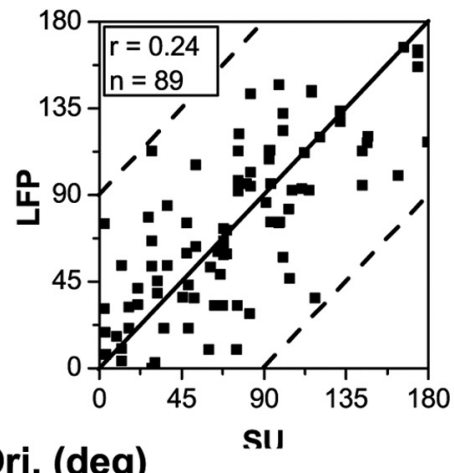

Opt. Carr. Ori. (deg)
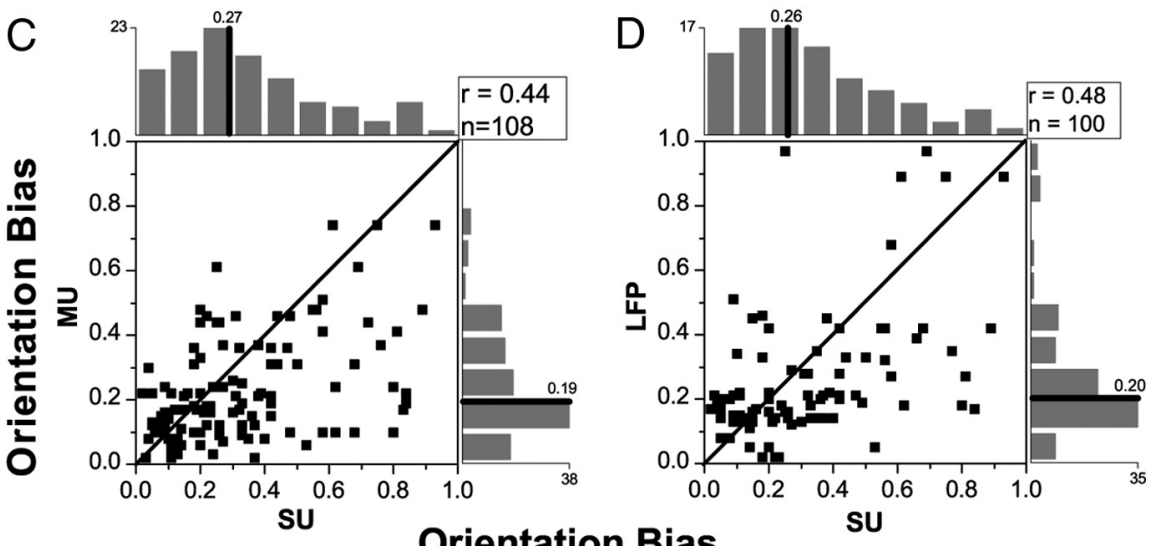

Orientation Bias

Figure 9. Comparison of carrier orientation tuning properties of simultaneously recorded MU_SU and LFP_SU pairs. In each around the diagonal line indicating a 1:1 ratio. $B$, Pairwise comparison of the optimal carrier orientations of LFP SU pairs. The data uning bandwidths of LFP_SU pairs. Most of the data points fall below the diagonal 1:1 line, indicating that, at most recording sites, the MU and LFP responses are less tuned than the SU responses. Lines in marginal histograms indicate median values.

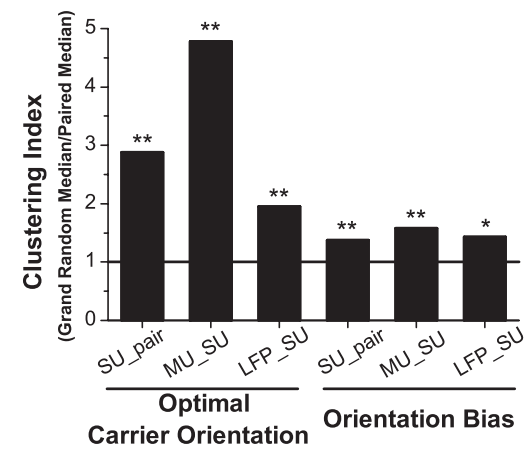

Figure 10. Quantitative analysis of clustering for carrier orientation tuning parameters, shown in same format as Figure 6 . Asterisks above some bars indicate that the associated clustering index is significantly $>1.0\left({ }^{*} p<0.05,{ }^{* *} p<0.01\right)$. None of these relationships were highly correlated. Horizontal line for clustering index, 1.0 .

$\mu \mathrm{m}$ ) but that carrier orientation may be arranged in a much more fine-grain manner.

\section{Discussion}

From comparisons of nearby SU, MU, and LFP signals across many recording sites, we have demonstrated that nearby neural signals showed very similar optimal carrier spatial frequency, indicating that it clearly exhibits a clustered organization. More- 


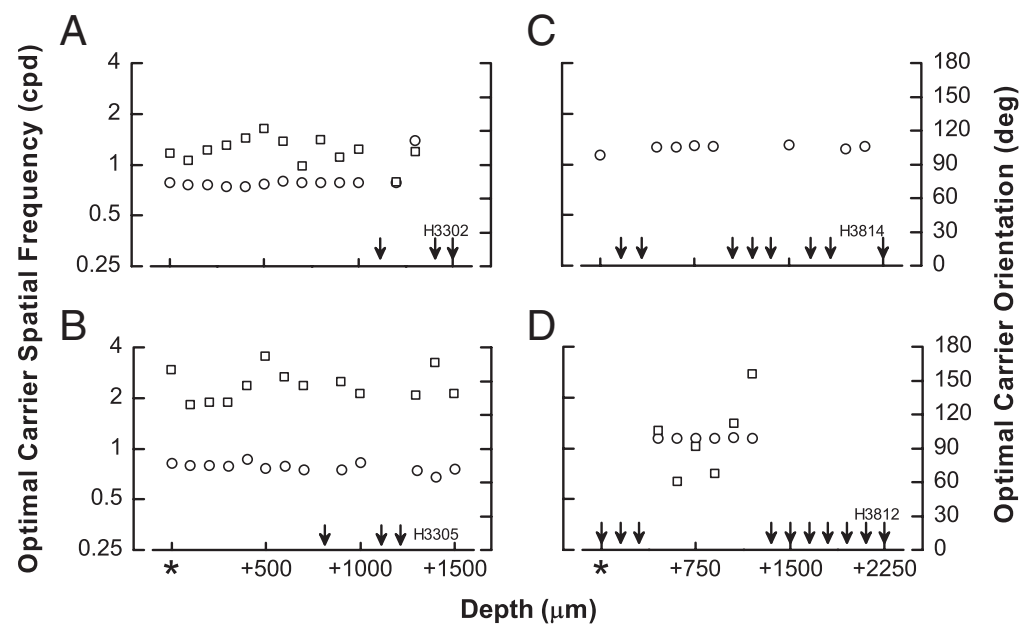

* 000000000009

$\rightarrow-100$ or $150 \mu \mathrm{m}$

Figure 11. Depth profiles of carrier spatial frequency and orientation tuning. Each panel shows an example of optimal carrier spatial frequencies and orientations extracted from recording sites along 16-channel linear array multielectrodes, in penetrations perpendicular to the cortical surface. Each data point indicates the optimal carrier spatial frequency (circle) or orientation (square) of the MU response extracted from one site along a linear array. The two sets of data shown in the left column $(\boldsymbol{A}, \boldsymbol{B})$ were recorded with an array having a site spacing of $100 \mu \mathrm{m}$ (covering a depth range of $1500 \mu \mathrm{m}$ ), whereas those shown in the right column (C, D) were recorded with an array having a $150 \mu \mathrm{m}$ site spacing (covering a depth range of $2250 \mu \mathrm{m}$ ). Arrows in each plot indicate sites at which MU activity was not CM responsive. Almost all CM-responsive sites along a given array exhibited similar optimal carrier spatial frequency, suggesting a columnar organization. However, carrier orientation results were more variable, with apparently rough constancy in $\boldsymbol{A}$ and $\boldsymbol{B}$ but not in $\boldsymbol{D}$ (results not obtained for dataset $\boldsymbol{C}$ ). ${ }^{*}$ denotes the first site of a linear array, and horizontal axis values indicate relative depths.

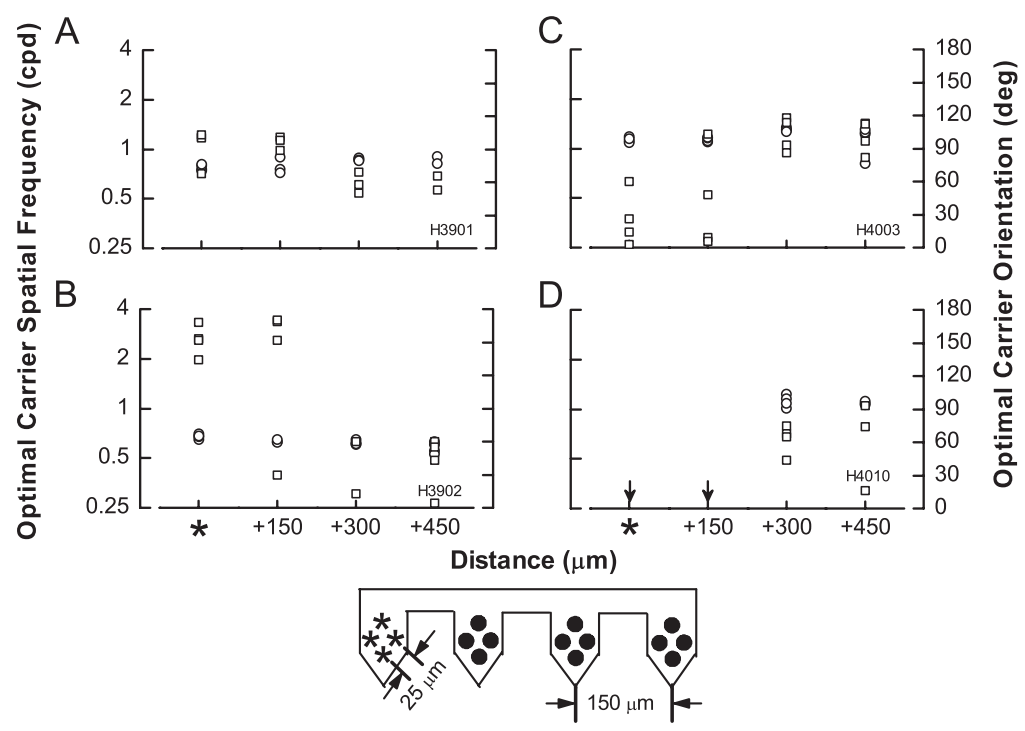

Figure 12. Four examples of optimal carrier spatial frequency and orientation extracted from recording sites across multishank tetrode arrays. Each data point indicates optimal spatial frequency (circles) or orientation (squares) of MU response extracted from one recording site in a tetrode of a four-shank tetrode array. The spacing between shanks is $150 \mu \mathrm{m}$, so the four shanks spanned a horizontal range of $450 \mu \mathrm{m}$. Arrows indicate sites that were not CM responsive. * denotes the first shank of four-shank tetrode array, with relative distances on the horizontal axis.

over, results from multielectrode arrays show that cells sensitive to the same carrier spatial frequency are organized in a columnar manner. The preferred carrier orientations of nearby neural signals were also significantly clustered, with a finer-grain patchy organization. Tuning bandwidth for carrier spatial frequency or orientation, conversely, did not show any significant indication of clustering. We also found that CM-responsive neurons were non-uniformly distributed across the volume of the cortical tissue.

\section{Columnar organization of carrier spatial frequency}

MU activity and LFPs are thought to sum responses from many neurons close to a recording site (Mitzdorf, 1987; Buzsáki, 2004; Liu and Newsome, 2006; Katzner et al., 2009; Xing et al., 2009) and have been demonstrated to reflect local columnar architecture in cat A17 (Katzner et al., 2009) and macaque MT (DeAngelis and Newsome, 1999; Liu and Newsome, 2003, 2006). Previous studies suggested that MU signals are detected over a range up to $\sim 65 \mu \mathrm{m}$ (Gray et al., 1995; Xing et al., 2009) and that the LFP sums cortical signals over a few hundred micrometers (Kruse and Eckhorn, 1996; Liu and Newsome, 2006; Berens et al., 2008) or, in later studies, $250 \mu \mathrm{m}$ (Katzner et al., 2009; Xing et al., 2009). If neurons exhibit similar tuning properties within a range that is smaller than or comparable with the range over which MU or LFP signals sum cortical activity, then these signals should exhibit similar tuning properties as simultaneously recorded SUs. For carrier spatial frequency, this was the case in our results. At most of our recording sites, the MU and LFP signals were clearly tuned to carrier spatial frequency with optimal values highly correlated with those of SUs, suggesting that neurons within a few hundred micrometers have similar carrier frequency tuning.

The similarity of optimal carrier spatial frequency along linear multielectrode arrays perpendicular to the brain surface (Fig. 11) implies a columnar organization. The similar optimal values across multishank tetrode arrays (Fig. 12) along the brain surface imply a columnar diameter of $\geq 450 \mu \mathrm{m}$. Together with the absence of $\mathrm{CM}$ response at some recording sites (see below), we infer that neurons tuned to similar optimal carrier spatial frequency form "detached patches" that are arranged in columns.

Our multielectrode recordings provide sufficient information to imply a columnar organization of carrier spatial frequency tuning, but they provide responses only across $1 \mathrm{~d}$ transects and therefore can only place limited constraints on the full 3 d layout of carrier spatial frequency clustering. The results suggest the existence of a carrier frequency "map" across the cortical surface but the small span and number of shanks on the tetrode arrays are insufficient to indicate the geometry of the mapping. Although the mapping of luminance spatial frequency has been visualized in cat A18 with optical imaging 
(Issa et al., 2000), the lack of correlation between carrier tuning properties and either envelope or luminance tuning (Zhou and Baker, 1996; Mareschal and Baker, 1998a) make it impossible to infer the layout of carrier spatial frequency columns by comparison with envelope or luminance results. Because the domains are on a scale of $\geq 450 \mu \mathrm{m}$, it might be profitable to use other kinds of multielectrode designs, or intrinsic signal optical imaging, to visualize the 2 d layout of carrier frequency tuning across the cortical surface.

\section{Structure of carrier orientation tuning properties}

The carrier orientation of nearby neurons was significantly clustered, but, unlike the carrier spatial frequency results, the clustering and pairwise linear correlations were less pronounced. The MU_SU pairs showed the highest clustering index and correlation coefficient for carrier orientation, whereas the LFP_SU pairs only exhibited modest values. The responses from multielectrode arrays often showed somewhat similar optimal values across adjacent recording sites, but by no means always, either along the brain surface or along sites perpendicular to the surface (Figs. 11, 12). Together with the pairwise clustering analysis, these results suggest that carrier orientation could be organized in "miniclusters," or possibly even forming "mini-columns," at a spatial scale comparable with that of MU signals $(65 \mu \mathrm{m})$ but less than that of LFP signals $(250 \mu \mathrm{m})$. In addition, some of our SU pairs exhibited very different carrier orientations, suggesting that mini-clusters having different optimal values may be tightly connected or partly overlapped or that there might be some random scatter in the organization.

Such a fine-grain clustering, together with the clear columnar organization of luminance orientation in this area (Hubel and Wiesel, 1965; Berman et al., 1987), implies that the optimal carrier orientation of a neuron would not be correlated with its optimal luminance orientation. This idea is consistent with SU studies (Mareschal and Baker, 1998a) demonstrating that there was no fixed relationship between the optimal carrier and envelope orientations of the neurons. Because carrier orientation appears to be organized as fine-grain mini-clusters, with substantial numbers of isotropic neurons, it would be difficult for intrinsic signal optical imaging to reveal its mapping. This may explain the failure to reveal carrier orientation maps in a recent study (Zhan and Baker, 2006). Other approaches are probably required to examine the actual size, shapes, and 3 d layout of these miniclusters, for example, two-photon imaging (Ohki et al., 2005).

\section{Non-uniform distribution of neurons responding to CM stimuli}

Previous SU studies demonstrated that only approximately half the sampled neurons in cat A18 (Zhou and Baker, 1996; Mareschal and Baker, 1999) and macaque V2 (von der Heydt et al., 1984) responded to second-order stimuli. Optical imaging (Ramsden et al., 2001; Zhan and Baker, 2006) suggested that these second-order responsive neurons are uniformly distributed across the cortical surface. However, optical imaging is of limited spatial resolution and mainly registers neural activity from the superficial layers. Thus, it is still unclear how second-order responsive neurons are arranged throughout the $3 \mathrm{D}$ volume of the cortex.

If the CM-responsive neurons were entirely uniform in anatomical distribution, then every recording site should be CM responsive for MU activity regardless of its location either across the surface or in depth. However, we frequently found sites that clearly responded to luminance stimuli but exhibited no response to CM stimuli along $1 \mathrm{D}$ profiles (Figs. 11, 12, arrows), suggesting that CM-responsive neurons might be non-uniformly distributed. However, because CM-responsive neurons are tuned to carrier spatial frequency and orientation, we must consider the possibility that we may have missed some neurons that were very narrowly tuned to particular combinations of carrier parameters and thus fell outside the limited number of stimulus conditions tested. This possibility is quite plausible for surface profiles (Fig. 12) but seems very unlikely along the depth profiles, because (as discussed above) CM-responsive sites along a linear array almost always had similar optimal carrier spatial frequency and orientation. Determining whether these unresponsive sites are randomly distributed or part of an orderly laminar organization will require a systematic laminar analysis incorporating anatomical registration.

\section{Functional organization of early visual cortex}

Our results demonstrate that neurons tuned to different carrier properties for second-order stimuli are neither uniformly nor randomly distributed but instead are clustered in a way that reflects a fine anatomical structure of cortical organization of second-order information processing. These results raise a number of questions for future research.

It is unclear from our results what is the full depth profile of responsivity to second-order stimuli and how it corresponds to anatomical laminae. Also, it remains to be seen how stimulus contrast affects the tuning properties for second-order stimuli and how this compares for LFP, MU, and SU signals and its laminar dependence.

The stronger clustering of carrier spatial frequency than of orientation could reflect a tradeoff between the number of mapped parameters and the decreasing unit size, representing specific feature combinations as the number of stimulus parameters increases (Swindale, 1998, 2000).

The finding of columnar organization suggests the opportunity to visualize coarse-scale cortical maps for carrier spatial frequency using intrinsic signal optimal imaging and to explore the micro-organization of very small-scale domains for carrier orientation, perhaps using two-photon imaging. Previous results have demonstrated maps for luminance orientation (Bonhoeffer and Grinvald, 1993), direction of motion (Shmuel and Grinvald, 1996), spatial frequency (Issa et al., 2000), and envelope orientation (Zhan and Baker, 2006) in cat area 18, and our results suggest at least one more, carrier spatial frequency, and possibly another, carrier orientation. This raises the questions of how carrier maps are organized on the cortical surface (Swindale, 2000), and what is their relationship to the other spatial maps. This in turn will provide a fresh opportunity to evaluate theoretical models of coverage uniformity (Swindale et al., 2000), wiring optimization (Chklovskii and Koulakov, 2004), constraints on how multiple parameters could be mapped (Swindale, 2004), and the relationships between them (Durbin and Mitchison, 1990).

\section{References}

Albright TD, Desimone R, Gross CG (1984) Columnar organization of directionally selective cells in visual area MT of the macaque. J Neurophysiol 51:16-31.

Berens P, Keliris GA, Ecker AS, Logothetis NK, Tolias AS (2008) Comparing the feature selectivity of the gamma-band of the local field potential and the underlying spiking activity in primate visual cortex. Front Syst Neurosci 2:2.

Berman NE, Wilkes ME, Payne BR (1987) Organization of orientation and direction selectivity in areas 17 and 18 of cat cerebral cortex. J Neurophysiol 58:676-699.

Bokil H, Andrews P, Kulkarni JE, Mehta S, Mitra PP (2010) Chronux: a platform for analyzing neural signals. J Neurosci Methods 192:146-151. 
Bonhoeffer T, Grinvald A (1993) The layout of iso-orientation domains in area 18 of cat visual cortex: optical imaging reveals a pinwheel-like organization. J Neurosci 13:4157-4180.

Brainard DH (1997) The psychophysics toolbox. Spat Vis 10:433-436.

Buzsáki G (2004) Large-scale recording of neuronal ensembles. Nat Neurosci 7:446-451.

Chklovskii DB, Koulakov AA (2004) Maps in the brain: what can we learn from them? Annu Rev Neurosci 27:369-392.

DeAngelis GC, Newsome WT (1999) Organization of disparity-selective neurons in macaque area MT. J Neurosci 19:1398-1415.

DeAngelis GC, Freeman RD, Ohzawa I (1994) Length and width tuning of neurons in the cat's primary visual cortex. J Neurophysiol 71:347-374.

DeAngelis GC, Ghose GM, Ohzawa I, Freeman RD (1999) Functional micro-organization of primary visual cortex: receptive field analysis of nearby neurons. J Neurosci 19:4046-4064.

Durbin R, Mitchison G (1990) A dimension reduction framework for understanding cortical maps. Nature 343:644-647.

Efron B, Tibshirani R (1993) An introduction to the bootstrap. New York: Chapman and Hall.

Fernald R, Chase R (1971) An improved method for plotting retinal landmarks and focusing the eyes. Vision Res 11:95-96.

Frien A, Eckhorn R, Bauer R, Woelbern T, Gabriel A (2000) Fast oscillations display sharper orientation tuning than slower components of the same recordings in striate cortex of the awake monkey. Eur J Neurosci 12:1453-1465.

Gieselmann MA, Thiele A (2008) Comparison of spatial integration and surround suppression characteristics in spiking activity and the local field potential in macaque V1. Eur J Neurosci 28:447-459.

Gray CM, Maldonado PE, Wilson M, McNaughton B (1995) Tetrodes markedly improve the reliability and yield of multiple single-unit isolation from multi-unit recordings in cat striate cortex. J Neurosci Methods 63:43-54.

Henrie JA, Shapley R (2005) LFP power spectra in V1 cortex: the graded effect of stimulus contrast. J Neurophysiol 94:479-490.

Hubel DH, Wiesel TN (1965) Receptive fields and functional architecture in two nonstriate visual areas (18 and 19) of the cat. J Neurophysiol 28:229-289.

Hubel DH, Wiesel TN (1974) Sequence regularity and geometry of orientation columns in the monkey striate cortex. J Comp Neurol 158:267-293.

Issa NP, Trepel C, Stryker MP (2000) Spatial frequency maps in cat visual cortex. J Neurosci 20:8504-8514.

Katzner S, Nauhaus I, Benucci A, Bonin V, Ringach DL, Carandini M (2009) Local origin of field potentials in visual cortex. Neuron 61:35-41.

Kayser C, König P (2004) Stimulus locking and feature selectivity prevail in complementary frequency ranges of V1 local field potentials. Eur J Neurosci 19:485-489.

Khayat PS, Niebergall R, Martinez-Trujillo JC (2010) Frequency-dependent attentional modulation of local field potential signals in macaque area MT. J Neurosci 30:7037-7048.

Kleiner M, Brainard D, Pelli D (2007) What's new in Psychtoolbox-3? Paper presented at the 36th European Conference on Visual Perception, Arezzo, Italy, August 27-31.

Kruse W, Eckhorn R (1996) Inhibition of sustained gamma oscillations $(35-80 \mathrm{~Hz}$ ) by fast transient responses in cat visual cortex. Proc Natl Acad Sci USA 93:6112-6117.

Leventhal AG, Wang Y, Pu M, Zhou Y, Ma Y (2003) GABA and its agonists improved visual cortical function in senescent monkeys. Science 300:812-815.

Liu J, Newsome WT (2003) Functional organization of speed tuned neurons in visual area MT. J Neurophysiol 89:246-256.

Liu J, Newsome WT (2006) Local field potential in cortical area MT: stimulus tuning and behavioral correlations. J Neurosci 26:7779-7790.

Mareschal I, Baker CL Jr (1998a) A cortical locus for the processing of contrast-defined contours. Nat Neurosci 1:150-154.

Mareschal I, Baker CL Jr (1998b) Temporal and spatial response to secondorder stimuli in cat area 18. J Neurophysiol 80:2811-2823.

Mareschal I, Baker CL Jr (1999) Cortical processing of second-order motion. Vis Neurosci 16:527-540.

Mitra PP, Bokil H (2008) Observed brain dynamics. New York: Oxford UP.
Mitzdorf U (1987) Properties of the evoked potential generators: current source-density analysis of visually evoked potentials in the cat cortex. Int J Neurosci 33:33-59.

Mountcastle VB (1997) The columnar organization of the neocortex. Brain 120:701-722.

Nauhaus I, Ringach DL (2007) Precise alignment of micromachined electrode arrays with V1 functional maps. J Neurophysiol 97:3781-3789.

Nikara T, Bishop PO, Pettigrew JD (1968) Analysis of retinal correspondence by studying receptive fields of binocular single units in cat striate cortex. Exp Brain Res 6:353-372.

Ohki K, Chung S, Ch'ng YH, Kara P, Reid RC (2005) Functional imaging with cellular resolution reveals precise micro-architecture in visual cortex. Nature 433:597-603.

Pelli DG (1997) The VideoToolbox software for visual psychophysics: transforming numbers into movies. Spat Vis 10:437-442.

Pesaran B, Pezaris JS, Sahani M, Mitra PP, Andersen RA (2002) Temporal structure in neuronal activity during working memory in macaque parietal cortex. Nat Neurosci 5:805-811.

Ramsden BM, Hung CP, Roe AW (2001) Real and illusory contour processing in area V1 of the primate: a cortical balancing act. Cereb Cortex 11:648-665.

Rosenberg A, Issa NP (2011) The y cell visual pathway implements a demodulating nonlinearity. Neuron 71:348-361.

Shmuel A, Grinvald A (1996) Functional organization for direction of motion and its relationship to orientation maps in cat area 18. J Neurosci 16:6945-6964.

Siegel M, König P (2003) A functional gamma-band defined by stimulusdependent synchronization in area 18 of awake behaving cats. J Neurosci 23:4251-4260.

Slepian D, Pollack HO (1961) Prolate spheroidal wave functions, Fourier analysis and uncertainty. I. Bell Syst Tech J 40:43-63.

Song Y, Baker CL Jr (2007) Neuronal response to texture- and contrastdefined boundaries in early visual cortex. Vis Neurosci 24:65-77.

Swindale NV (1998) Cortical organization: modules, polymaps and mosaics. Curr Biol 8:R270-R273.

Swindale NV (2000) How many maps are there in visual cortex? Cereb Cortex 10:633-643.

Swindale NV (2004) How different feature spaces may be represented in cortical maps. Network 15:217-242.

Swindale NV, Shoham D, Grinvald A, Bonhoeffer T, Hübener M (2000) Visual cortex maps are optimized for uniform coverage. Nat Neurosci 3:822-826.

Tanaka H, Ohzawa I (2006) Neural basis for stereopsis from second-order contrast cues. J Neurosci 26:4370-4382.

Thomson DJ (1982) Spectrum estimation and harmonic-analysis. Proc IEEE 70:1055-1096.

Tusa RJ, Rosenquist AC, Palmer LA (1979) Retinotopic organization of areas 18 and 19 in the cat. J Comp Neurol 185:657-678.

Viswanathan A, Freeman RD (2007) Neurometabolic coupling in cerebral cortex reflects synaptic more than spiking activity. Nat Neurosci 10:1308-1312.

von der Heydt R, Peterhans E, Baumgartner G (1984) Illusory contours and cortical neuron responses. Science 224:1260-1262.

Wörgötter F, Eysel UT (1987) Quantitative determination of orientational and directional components in the response of visual cortical cells to moving stimuli. Biol Cybern 57:349-355.

Xing D, Yeh CI, Shapley RM (2009) Spatial spread of the local field potential and its laminar variation in visual cortex. J Neurosci 29:11540-11549.

Yao H, Dan Y (2001) Stimulus timing-dependent plasticity in cortical processing of orientation. Neuron 32:315-323.

Zhan CA, Baker CL Jr (2006) Boundary cue invariance in cortical orientation maps. Cereb Cortex 16:896-906.

Zhou YX, Baker CL Jr (1993) A processing stream in mammalian visual cortex neurons for non-Fourier responses. Science 261:98-101.

Zhou YX, Baker CL Jr (1994) Envelope-responsive neurons in areas 17 and 18 of cat. J Neurophysiol 72:2134-2150.

Zhou YX, Baker CL Jr (1996) Spatial properties of envelope-responsive cells in area 17 and 18 neurons of the cat. J Neurophysiol 75:1038-1050. 